\title{
Ekman-Inertial Instability
}

By

Nicolas Grisouard (nicolas.grisouard@utoronto.ca) and

Varvara E. Zemskova (barbara.zemskova@utoronto.ca)

University of Toronto, Department of Physics, 60 St. George Street, Toronto ON M5S 1A7, Canada

This paper is a peer-reviewed preprint submitted to EarthArXiv. It was submitted to Physical Review Fluids on 26 June 2020 and accepted on 16 November 2020. Once production is complete, the final version of this manuscript will be available via the 'Peer-review Publication DOI' link on the right-hand-side of this webpage. Please feel free to contact any of the authors. 


\section{Ekman-Inertial Instability}

Nicolas Grisouard斗 and Varvara E. Zemskova

University of Toronto, Department of Physics, 60 St. George Street, Toronto ON M5S 1A7, Canada

(Dated: November 18, 2020) 
We report on an instability, arising in sub-surface, laterally-sheared geostrophic flows. When the lateral shear of a horizontal flow in geostrophic balance is of opposite sign to the Coriolis parameter, and exceeds it in magnitude, embedded perturbations are subjected to inertial instability, albeit modified by viscosity. When the perturbation arises from the surface of the fluid, the initial response is akin to a Stokes problem, with an initial flow aligned with the initial perturbation. The perturbation then grows quasi-inertially, rotation deflecting the velocity vector, which adopts a well-defined angle with the mean flow $\{$, and viscous stresses transferring horizontal momentum downward. The combination of rotational and viscous effects in the dynamics of inertial instability prompts us to call this process "Ekman-inertial instability". While the perturbation initially grows super-inertially, the growth rate then becomes sub-inertial, eventually tending back to the inertial value. The same process repeats downward as time progresses.

Ekman-inertial transport aligns with the asymptotic orientation of the flow, and grows exactly inertially with time, once the initial disturbance has passed. Because of the strongly super-inertial initial growth rate, this instability might compete favorably against other instabilities arising in ocean fronts.

\section{INTRODUCTION}

When wind blows over the ocean surface over long periods of time, momentum diffuses down in a very different manner from Stokes' first problem. Instead, the Coriolis acceleration balances downward diffusion of momentum to form Ekman spirals [1]. According to its simplest description [2], horizontal velocity at the surface forms a $45^{\circ}$-angle with the direction of the wind, and within the Ekman layer (hereafter referred to as EL), spirals down to zero over a depth $\sim \sqrt{2 \nu / f}$, where $\nu$ is the kinematic viscosity (hereafter "viscosity"), in practice the vertical eddy viscosity, and $f$ is the Coriolis parameter. In spite of its simplicity and notorious difficulty to directly observe in the ocean, this solution has allowed some significant advances in our understanding of ocean dynamics. For example, the predicted cumulative mass transport of ELs provides a relatively accurate explanation of how winds set up ocean gyres [1, 3, and references therein]. Since then, Ekman layer theory has been amended to

\footnotetext{
*nicolas.grisouard@utoronto.ca
} 
include weak vorticity effects [4] 6], or variability of the wind and eddy diffusivity both in space [7, time [8], or other features of the upper ocean [9].

EL theory has seen a renewed interest in the context of submesocale studies [10, 11]. Submesoscale flows are defined by a vertical vorticity field $\zeta=(\boldsymbol{\nabla} \times \boldsymbol{v}) \cdot \hat{\boldsymbol{z}}$ with magnitude comparable to the planetary vorticity $f$, i.e., Rossby number of order unity $($ Ro $=\zeta / f=O(1))$ [12] Near the ocean surface, submesoscale flows and their associated vertical velocities could be important for ecosystems [13 15], atmosphere-ocean exchanges [16, 17], or as a kinetic energy sink that could help, closing the energy budget of the ocean [11, 18]. Recent studies have expanded our understanding of submesoscale ELs and their impacts by incorporating interactions with $\mathrm{Ro}=O(1)$ vortical flows [3], surface waves and Langmuir circulation [9, 19], and modifications due to baroclinic pressure torques [9, 16, 20, 21].

In the present study, our goal is to contribute to this effort by describing what we hereafter refer to as "Ekman-Inertial Instability" (EII), which can be seen as the unstable counterpart of an EL that occurs in anticyclonic flows for which Ro $<-1$. In the oceanic regime, and independently of the results we are about to present, such flows can undergo inertial instability (InI), in which a particle slightly displaced across a geostrophic jet will find itself in a region where the imbalance between ambient pressure gradient and the Coriolis force tends to amplify its displacement [22, 23]. The main features of InI are well-described by linear stability analysis, i.e., by the growth of a plane wave-like mode at a rate of $f \sqrt{-1-\text { Ro }}$ in the inviscid limit, constant in time and space.

EII, on the other hand, originates from a change in wind stress at the surface of the ocean, and the vertical extent over which it impacts the fluid increases downward due to viscous stresses, eventually following a typical $\sqrt{\nu t}$ scaling. When Ro $<-1$, it replaces the Ekman layer spin-up, which occurs for Ro $>-1$. In a first phase, which we will refer to as "viscous-inertial peeling", tangential viscous stresses act to set the fluid in motion much faster than the expected exponential growth of InI. In this first phase, the problem is mathematically equivalent to Stoke's first (or Rayleigh) problem, albeit for the vertical shear. In particular, in the case of a sudden wind change, it inherits its initially infinite growth rate. Past this initial phase, the flow keeps accelerating in a quasi-exponential manner and draws its energy from the lateral shear of the geostrophic current, akin to InI, albeit slowed down by downward diffusion of momentum by viscosity. Originating at the surface, these processes repeat at later times at greater depths. 
In the next section, we derive the expressions of the velocity field under EII, followed in $\S$ III by a description of how EII physically manifests itself. In $\S$ IV] we discuss how EII would insert itself in the dynamical landscape of an unstable front and in particular, we compare EII with the classical theory of InI in order to predict how they would compete, and attempt to predict how EII would play out in a front of finite width. Finally, we offer a summary and conclusions in $\S \mathrm{V}$.

\section{MATHEMATICAL DESCRIPTION}

We present here the solution for the most idealized version of EII. We mirror this derivation with its "stable" counterpart, i.e., the establishment of an EL accompanied by nearinertial oscillations, in the Appendix.

\section{A. Posing the problem}

We start from the equations of motion of an incompressible, homogeneous flow, with a traditional $f$-plane approximation, i.e.,

$$
\tilde{\boldsymbol{v}}_{t}+\tilde{\boldsymbol{v}} \cdot \boldsymbol{\nabla} \tilde{\boldsymbol{v}}+f \hat{\boldsymbol{z}} \times \tilde{\boldsymbol{v}}+\nabla \tilde{p} / \rho=\nu \nabla^{2} \tilde{\boldsymbol{v}} \quad \text { and } \quad \nabla \cdot \tilde{\boldsymbol{v}}=0
$$

where $\tilde{\boldsymbol{v}}=(\tilde{u}, \tilde{v}, \tilde{w})$ is the full velocity field in a direct Cartesian coordinate system $(\hat{\boldsymbol{x}}, \hat{\boldsymbol{y}}, \hat{\boldsymbol{z}})$, with $\hat{\boldsymbol{z}}$ pointing upward. Subscripts denote partial derivatives, $\tilde{p}$ the deviations from hydrostatic pressure, and $\rho$ the constant fluid density.

We next decompose our flow into a component, denoted by bars, that flows in the $y$ direction and is in geostrophic balance with the pressure force in the $x$-direction, and deviations from it, namely,

$$
\tilde{\boldsymbol{v}}=\bar{v}(x, z) \hat{\boldsymbol{y}}+(u, v, w) \quad \text { and } \quad \tilde{p}=\bar{p}+p \quad \text { such that } \quad f \bar{v}=\bar{p}_{x} / \rho
$$

The geostrophic balance above neglects viscous diffusion of momentum, which we justify by assuming that the spatial scales of the geostrophic flow are too large for it to act over the time scales of EII. We let the velocity vary in the across-jet direction, which defines a local Rossby number

$$
\operatorname{Ro}=\bar{v}_{x} / f .
$$


We treat Ro as a constant, i.e., we focus on the case of linear lateral shear for $\bar{v}$ : a strong simplification in the submesoscale regime, but one that captures the essential physics of EII. Note that this assumption enforces geostrophic current's expressions of the form $\bar{v}(x, z)=$ $f \operatorname{Ro} x+\varphi(z)$, where $\varphi$ is a function of $z$ only. Also note that by treating Ro as a constant, we are effectively setting up an infinite reservoir of energy, EII can grow from. We then complete our initial set-up by adding boundary conditions at the surface, located at $z=0$, namely, a rigid lid and an initial wind stress in the $y$-direction only, defined as $T_{I}^{y}=\left.\rho \nu \bar{v}_{z}\right|_{z=0}$, such that $\tilde{\boldsymbol{v}}=\bar{v} \hat{\boldsymbol{y}}$ is a steady solution of our initial system (1) and the boundary conditions above. The deviations from this initial state, i.e., $u, v, w$ and $p$, are initially zero. A change (i.e., an increase, decrease, and/or change of direction) of the wind stress, starting at $t=0$, will initiate EII.

\section{B. EII derivation}

Like in ELs and InI, a constant Ro allows us to ignore all of the horizontal derivatives in the $u, v, w$ and $p$ fields. Doing so, along with using the incompressibility (1) and the top rigid lid conditions, yields $w \equiv 0$. Collecting everything, the only remaining advective term in the momentum equations (1) is $\tilde{u} \tilde{v}_{x} \hat{\boldsymbol{y}}=f$ Rou $\hat{\boldsymbol{y}}$, while all others are exactly zero. The equations of motion (1) then reduce to

$$
u_{t}-f v=\nu u_{z z} \quad \text { and } \quad v_{t}+(1+\mathrm{Ro}) f u=\nu v_{z z}
$$

with the other components of eqn. (1) being trivially satisfied.

EII starts at $t=0$ with wind stress that evolves as $T^{y}(t)$, which translates into the following boundary condition for the deviations:

$$
\left.v_{z}\right|_{z=0}=a(t)=\left[T^{y}(t)-T_{I}^{y}\right] /(\nu \rho) \text { for } \quad t>0 .
$$

EL boundary conditions close the system, i.e.,

$$
\left.u_{z}\right|_{z=0}=0 \text { and } \lim _{z \rightarrow-\infty}(u, v)=0 .
$$

Note that we could include a wind stress in the $x$-direction at a relatively modest analytical cost. The solution would only change quantitatively, and the expressions of the solution would be almost the same as the ones we are about to derive (not shown). 
Classically, i.e., for $\operatorname{Ro}>-1$, we would see a transient adjustment including the radiation of near-inertial waves and/or the spin-up of an EL if $T^{y}(t)$ were to reach a constant value (we explicitly compute such a case in the Appendix). However, for Ro $<-1$, EII replaces this adjustment, and does not feature either waves or an EL. Instead, as we will show, the flow will grow monotonically.

In order to decouple eqns. (44), we introduce

$$
U=u+v / \alpha \quad \text { and } \quad V=-u+v / \alpha,
$$

with $\alpha^{2}=-1-$ Ro. In scaled coordinates

$$
\tau=F t \quad \text { and } \quad Z=z / \delta,
$$

where $F=\alpha f$ and $\delta=\sqrt{2 \nu / F}$, eqns. (4) become

$$
U_{\tau}-U=U_{Z Z} / 2 \text { and } V_{\tau}+V=V_{Z Z} / 2 .
$$

Introducing $U^{\dagger}=U \mathrm{e}^{-\tau}$ in the first equation above reduces it to the mere diffusion equation

$$
2 U_{\tau}^{\dagger}=U_{Z Z}^{\dagger} \quad \text { and }\left.\quad U_{Z}^{\dagger}\right|_{Z=0}=A(\tau) \mathrm{e}^{-\tau},
$$

with $A(\tau)=a(\tau) \delta / \alpha$, together with boundary conditions (6). The solution to this system is

$$
U^{\dagger}=\int_{0}^{\tau} \frac{A\left(\tau^{\prime}\right) \mathrm{e}^{-\tau^{\prime}}}{\sqrt{2 \pi\left(\tau-\tau^{\prime}\right)}} \exp \left(-\frac{Z^{2}}{2\left(\tau-\tau^{\prime}\right)}\right) \mathrm{d} \tau^{\prime} .
$$

After multiplying with $\mathrm{e}^{\tau}$ and the change of variables $\theta \mapsto \tau-\tau^{\prime}$,

$$
U=\int_{0}^{\tau} \frac{A(\tau-\theta)}{\sqrt{2 \pi \theta}} \exp \left(\theta-\frac{Z^{2}}{2 \theta}\right) \mathrm{d} \theta .
$$

A similar derivation, using $V^{\ddagger}=V \mathrm{e}^{\tau}$ instead of $U^{\dagger}=U \mathrm{e}^{-\tau}$ in eqn. (9), yields

$$
V=\int_{0}^{\tau} \frac{A(\tau-\theta)}{\sqrt{2 \pi \theta}} \exp \left(-\theta-\frac{Z^{2}}{2 \theta}\right) \mathrm{d} \theta,
$$

from which we can deduce the solutions to the original eqns. (4), namely

$$
\left[\begin{array}{l}
u \\
v
\end{array}\right]=\frac{1}{2}\left[\begin{array}{c}
U-V \\
\alpha(U+V)
\end{array}\right]=\int_{0}^{\tau} \frac{A(\tau-\theta)}{\sqrt{2 \pi \theta}}\left[\begin{array}{c}
\sinh \theta \\
\alpha \cosh \theta
\end{array}\right] \exp \left(-\frac{Z^{2}}{2 \theta}\right) \mathrm{d} \theta .
$$

The expressions above do not make it immediately clear that the flow represents an instability. This fact will become apparent in the step response to a surface disturbance, which we will derive after we introduce our numerical validation strategy. 


\section{Validation strategy}

To independently validate our findings, we solve equations (40)-(6) in the case of an abrupt change in boundary conditions (constant $a$ and $A$ ) with the spectral code Dedalus 24 [25]. The depth of our domain is $15 \delta$, and we use 256 Chebyshev modes. We integrate the equations over $15 / F$, which is long enough to see EII mature, but short enough that it does not reach the bottom of the domain, in agreement with the condition at infinity in eqns. (6). Because the one-dimensional equations (4) are linear, Dedalus integrates them implicitly in time with a $4^{\text {th }}$-order Runge-Kutta scheme. At the start of the simulation, $u$ and $v$ vary more strongly. To account for it, we progressively increase the time step from $10^{-5} \mathrm{~F}^{-1}$ in the beginning, to $10^{-2} \mathrm{~F}^{-1}$ at infinity, over a duration $F^{-1}$. However, we did not attempt to optimize the time steps because the integrations complete within seconds on a personal computer.

Simulations shown here are seeded with noise, meaning that EII and InI compete. However, noise-free simulations (not shown) behave virtually identically. As expected from linear calculations, outcomes of numerical simulations and analytical solutions are practically indistinguishable. We present both below for abrupt wind change.

\section{Solution following an abrupt wind change}

We now focus on the case when wind starts abruptly, i.e., for constant $A(\tau)=A_{0}$. Note that eqns. (10) are formally identical to Stoke's first (or Rayleigh) problem for $U_{Z}^{\dagger}$. Therefore, any change in wind stress will imply an infinitely fast adjustment of the vertical shear at the surface, which will later translate into an initially infinite growth rate of EII. Physically speaking, it means that EII will initially respond as fast as the wind evolves, before taking on a life of its own. We numerically tested moderate departures from this case, e.g., exponential approach to different, constant wind stress values over time scales similar to $1 / F$ or shorter and found qualitatively and quantitatively similar behavior to the abrupt change case. Should the wind evolve over longer time scales, EII would likely initiate and saturate before said time scales have time to impart their signature on the flow. 

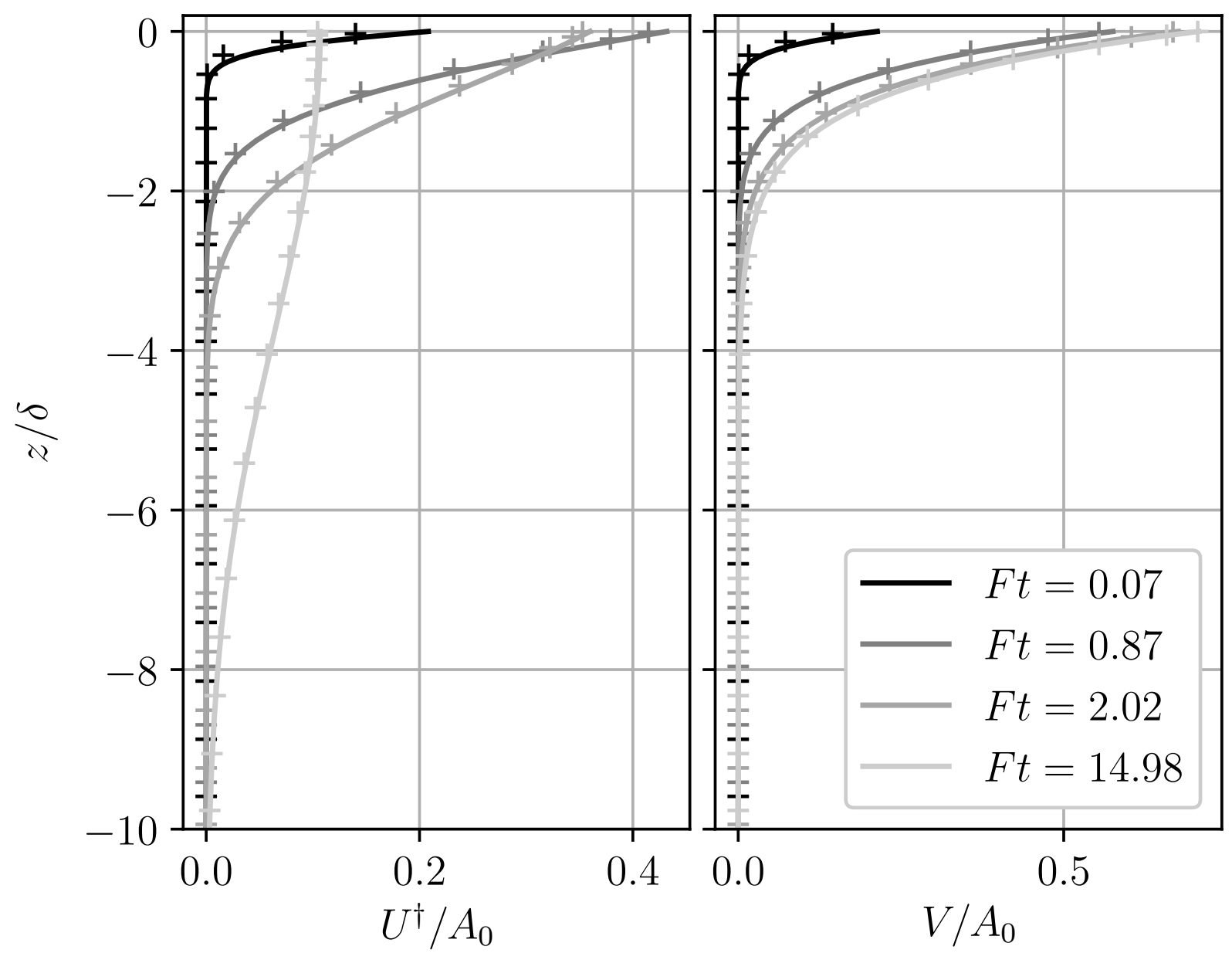

FIG. 1. Evolution of the profiles of $U^{\dagger}$ and $V$, after an abrupt change in boundary conditions. Solid lines: analytical solutions derived in $\S$ IID ; crosses: independent numerical integration of eqns. (4), described in $\S$ II C. We only display one cross every eight grid points.

Under this condition, eqn. (12) can be cast in the following closed forms

$$
\begin{aligned}
U & =\frac{A_{0}}{\sqrt{2}} \Im\left[\mathrm{e}^{Z i \sqrt{2}} \operatorname{erfc}\left(-i \sqrt{\tau}-\frac{Z}{\sqrt{2 \tau}}\right)\right] \\
& =\frac{A_{0}}{\sqrt{2}} \mathrm{e}^{\tau-Z^{2} /(2 \tau)} \Im\left[\mathcal{W}\left(\sqrt{\tau}+\frac{i Z}{\sqrt{2 \tau}}\right)\right],
\end{aligned}
$$

where erfc is the complementary error function, $\Im$ denotes the imaginary part and $\mathcal{W}$ is the Faddeeva function

$$
\forall \xi \in \mathbb{C}, \quad \mathcal{W}(\xi)=\mathrm{e}^{-\xi^{2}} \operatorname{erfc}(-i \xi)
$$

We plot $U^{\dagger}$ corresponding to this solution in figs. 1] (left panel) and 2 (top panel).

Eqn. (15b) highlights the long-term behavior of the solution. First, $\mathrm{e}^{\tau}$ is the only factor 


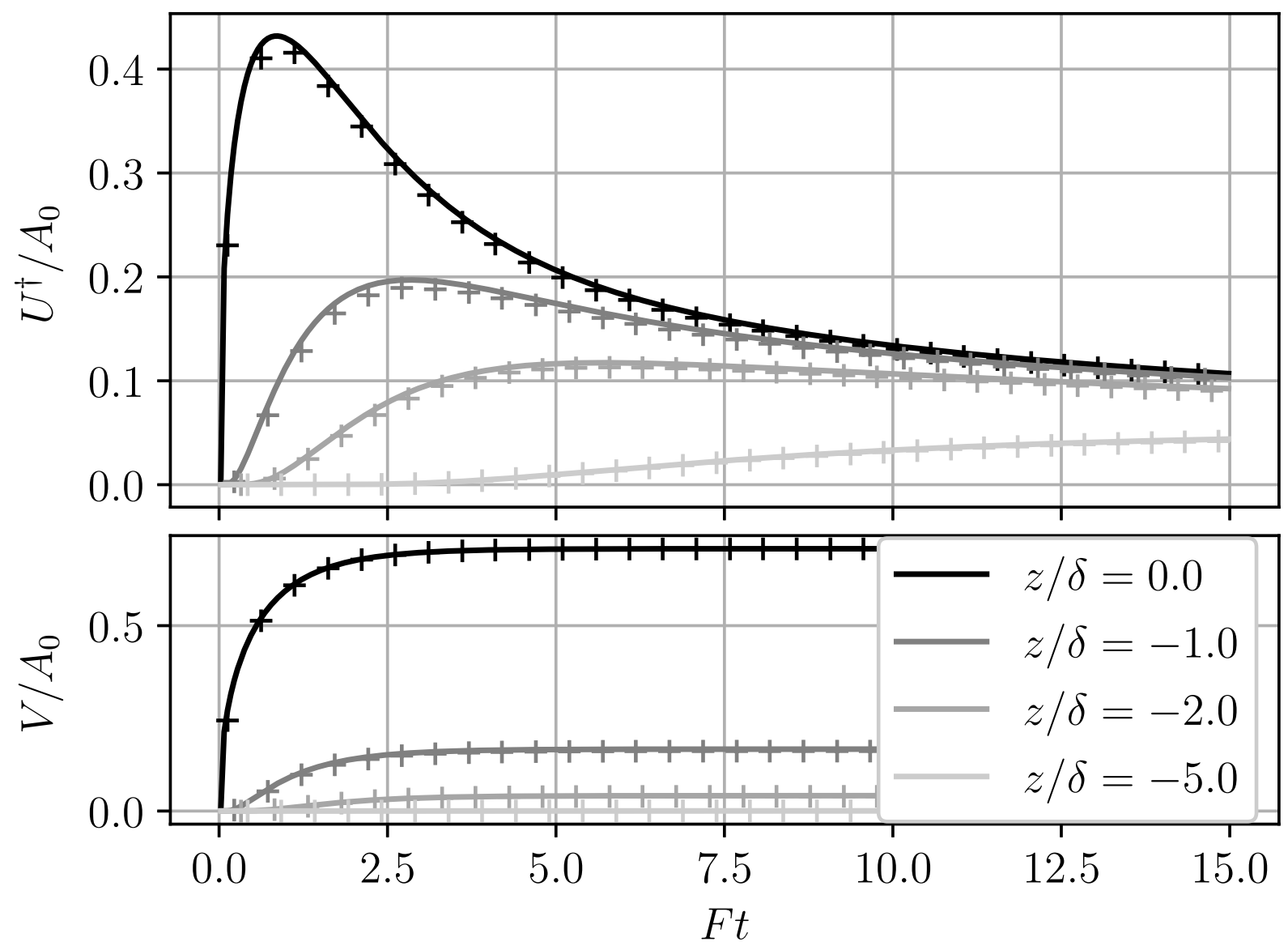

FIG. 2. Same as fig. 1 presented as time series at a few depths. We only display one cross every ten time steps.

that exhibits a persistently growing behavior, while the rest, namely, $U^{\dagger}$, is bounded at all times, which is why we only plot the latter in figs. 1 and 2 . In fact, $U$ grows indefinitely, albeit at a rate that keeps evolving, which we will discuss in $\S \amalg \mathrm{I}$. Second, for $\tau \gg 1$, $\mathcal{W}(\ldots) \approx \mathcal{W}(\sqrt{\tau})$, and the $Z$-dependence mostly manifest itself in the $\mathrm{e}^{-Z^{2} /(2 \tau)}$ factor. Therefore, the bell-shaped profile of $U^{\dagger}$ found at $F t \approx 15$ in fig. 1 is a weakly-modulated Gaussian, whose vertical extent scales as $\sqrt{\nu t}$ in dimensional coordinates.

Similarly, eqn. (13) becomes

$$
V=\frac{A_{0}}{2 \sqrt{2}}\left[\mathrm{e}^{Z \sqrt{2}} \operatorname{erfc}\left(-\sqrt{\tau}-\frac{Z}{\sqrt{2 \tau}}\right)-\mathrm{e}^{-Z \sqrt{2}} \operatorname{erfc}\left(\sqrt{\tau}-\frac{Z}{\sqrt{2 \tau}}\right)\right],
$$

which we plot in figs. 1 (right panel) and 2 (bottom panel). Contrary to $U$, the error functions above have real arguments, bounding $V$ at all times and depths. In particular, for $\tau \gg 1, V \approx A_{0} / \sqrt{2} \mathrm{e}^{Z \sqrt{2}}$ and does not extend deeper than $O(\delta)$. 
Figs. 1 and 2 show that EII is most pronounced at the surface. There, eqns. (15) have simple analytical expressions, namely

$$
\begin{aligned}
& \left.U\right|_{Z=0}=\frac{A_{0}}{\sqrt{2}} \operatorname{erfi}(\sqrt{\tau})=\sqrt{\frac{2}{\pi}} A_{0} \mathrm{e}^{\tau} \mathrm{D}(\sqrt{\tau}), \\
& \left.V\right|_{Z=0}=\frac{A_{0}}{\sqrt{2}} \operatorname{erf}(\sqrt{\tau}),
\end{aligned}
$$

where erfi is the imaginary error function and

$$
\forall \xi \in \mathbb{R}, \quad \mathrm{D}(\xi)=\frac{\sqrt{\pi}}{2} \mathrm{e}^{-\xi^{2}} \operatorname{erfi}(\xi)
$$

is the Dawson integral. The latter is bounded, with $\mathrm{D}(\sqrt{\tau}) \approx \sqrt{\tau}$ for $\tau \ll 1$, then going though a maximum at $\tau \approx 0.92$, before decaying monotonically to zero, eventually as $1 /(2 \sqrt{\tau})$

\section{E. Growth rate}

The general expression for the growth rate of $U$ is

$$
\sigma_{U}(t, Z)=\frac{1}{U} \frac{\partial U}{\partial t}=F+\frac{1}{U^{\dagger}} \frac{\partial U^{\dagger}}{\partial t}
$$

We hereafter refer to periods of time when $\sigma_{U}>F\left(\sigma_{U}<F\right)$ as "super-inertial" ("subinertial"), in reference to the growth rate of inviscid InI.

The growth rates of $U$ and $V$ can be readily obtained from eqns. (12)-13 and the Leibniz integral rule. We explicitly plot $\sigma_{U}$ in the case of a sudden wind change in fig. 3 . In accordance with eqn. (18), periods of $U^{\dagger}$ increasing (decreasing) in fig. 2 correspond to phases over which $U$ grows super-inertially (sub-inertially). Qualitatively, the growth rate behaves similarly at all depths. Thus, we focus on the surface behavior, which also has the strongest impact on the dynamics of a front. There,

$$
\sigma_{0}=\left.\sigma_{U}\right|_{Z=0}=[2 \sqrt{\tau} \mathrm{D}(\sqrt{\tau})]^{-1},
$$

which we can break down following the discussion at the end of $\S$ IID. That is, for $\tau \ll 1$, $\sigma_{0} \approx 1 /(2 \tau)$, and the growth rate goes from infinity to unity within a duration $\tau \approx 0.854$. It then decreases and reaches a minimum of $\sigma_{0} \approx 0.778 F$ at $\tau \approx 2.26$. The growth rate then monotonically increases and asymptotically tends to $F$. 


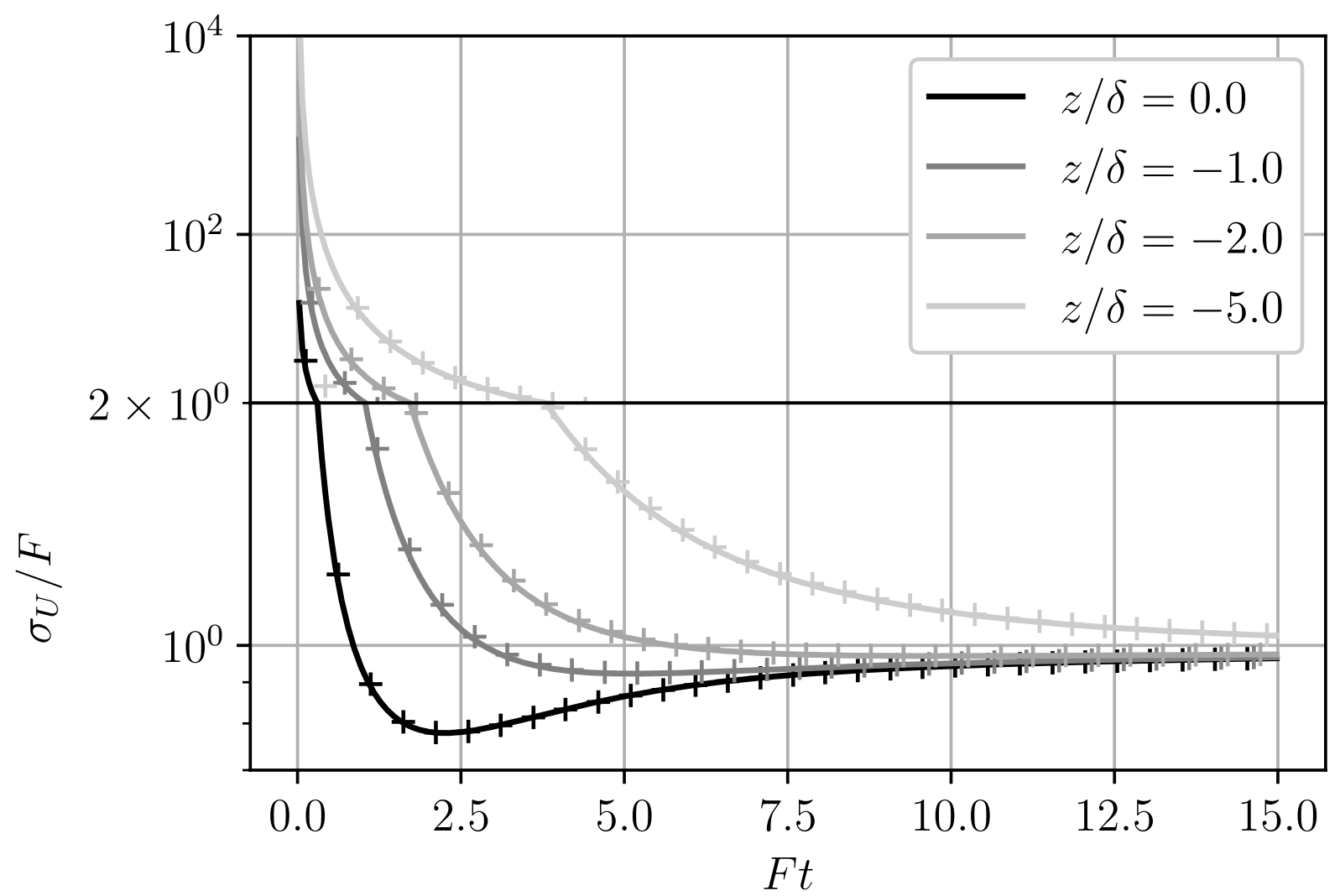

FIG. 3. Same as fig. 2, for growth rates $\sigma_{U}$. Note the change in vertical log scale at $\sigma_{U}=2 F$, curves would appear inifintely differentiable otherwise.

At depth, the flow qualitatively goes through the same series of steps, with quantitative differences. As $Z$ decreases, the initial growth rate increases in absolute value due to lower values of $U$. It reaches the $\sigma_{U}=F$ mark, then its minimum value, which is closer to $F$ at greater depth, at later times.

\section{INSTABILITY DYNAMICS}

This section presents a more qualitative description of EII, namely, the physical mechanisms involved, the morphology of the induced flow, and the implication on mass transport. 


\section{A. Dynamics through the lens of energetics}

The individual mechanisms involved in EII can be better traced by investigating their energetic signatures. From eqns. (4), the evolution equation of the kinetic energy density of the flow $K=\left(u^{2}+v^{2}\right) / 2$ is

$$
K_{t}=-L S P-\Phi_{z}-\varepsilon,
$$

where $L S P=$ Rofuv stands for Lateral Shear Production, i.e., the transfer of kinetic energy from perturbations to the mean shear (negative here); $\Phi=-\nu K_{z}$, the viscous diffusive flux of kinetic energy; and $\varepsilon=\nu\left(u_{z}^{2}+v_{z}^{2}\right)$, the irreversible dissipation.

Fig. (4) shows that $\Phi_{z}$ plays a role that depends on the phase of EII. In the first phase, which we refer to as "Viscous-Inertial Peeling" (VIP), $-\Phi_{z}$ is the dominant energy source at the leading edge of the instability, setting the fluid in motion, with $-L S P$ being the secondary energy source. This phase (fig. 4, right panel) coincides with the super-inertial growth we described in $\S$ IIE. Near the surface, it lasts $O\left(F^{-1}\right)$, too short for rotation to influence the dynamics significantly. VIP is therefore a Rayleigh-like problem, rotation acting as a perturbation.

After the instability front has passed however (fig. $4, z / \delta>-1.5$ ), $-L S P$ becomes the dominant source of energy, as in InI, and $-\Phi_{z}$ acts to reduce the growth of the instability. Physically, rotation is now acting and the flow set in motion during VIP is inertially unstable, a phase we call "Inertial-Viscous Instability" (InVI). Upper layers of the fluid have begun going unstable earlier than lower layers, and their velocity proceeds to grow quasiexponentially. The result is a persistent horizontal momentum imbalance between upper and lower layers, which viscosity diffuses downward. InVI therefore behaves like a viscouslydragged InI. As time progresses, EII behaves more and more like inviscid InI: relatively speaking, the vertical gradients diminish (see fig. 1), $\Phi_{z}$ becomes less important, and the growth rate approaches $F$.

\section{B. Hodograph}

EII induces a peculiar velocity field, with some features reminiscent of the Ekman spiral (see fig. 5), with a caveat that we address in the next paragraph. During the early phases of VIP and near the surface, $\mathrm{e}^{ \pm \tau} \approx 1$ and eqns. 12 - 13 show that $U$ and $V$ both initially 

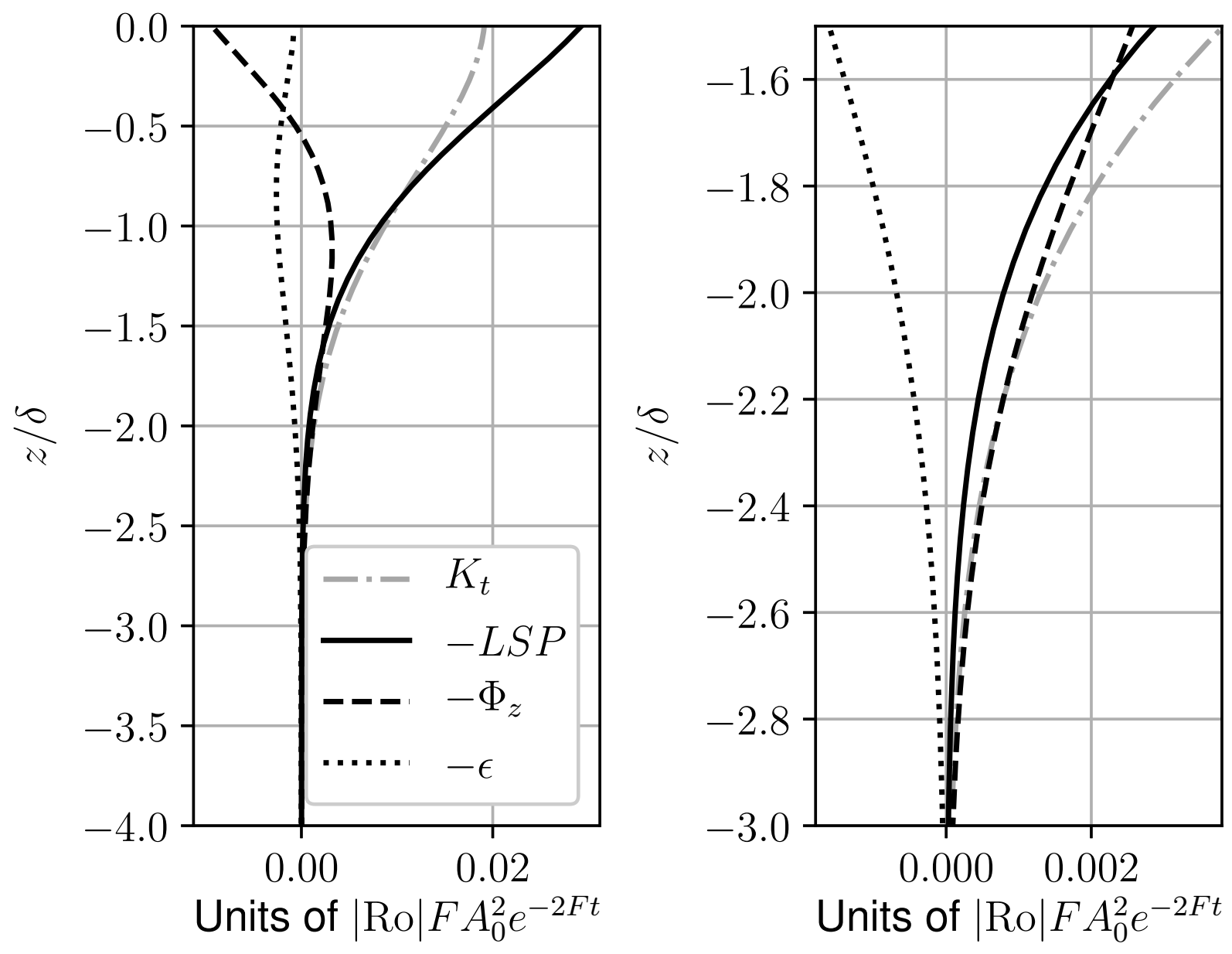

FIG. 4. Kinetic energy budget at $F t=2$ (cf. eqn. 20). Both panels display the same data, only the right-hand-side panel is a magnification of the left-hand-side panel around the edge of the EII propagation. These plots are of the numerical simulation.

grow at similar rates. Rotation is not acting yet, and the motion is along the original wind perturbation direction (fig. 5, left panel). Later, as VIP transitions into InVI near the surface, $V$ settles to a constant value, while $U$ keeps growing quasi-exponentially (recall $\S$ IID). The near-surface velocity vector therefore adopts an angle of $45^{\circ}$ with the mean flow in $u, v / \alpha$ coordinates (fig. 5, right panel). For $Z \ll-1$ however, $V \approx 0$ at all times, and the velocity vector adopts this $45^{\circ}$-angle immediately (fig. 5 , $z=-5 \delta$ lines).

We caution however on the analogy with ELs: the angle we just mentioned is with the direction of the mean flow, not that of the wind direction. Indeed, the appearance of this angle traces its roots back to eqns. (9), and to $U$ and $V$ being the solution of an unstable and 

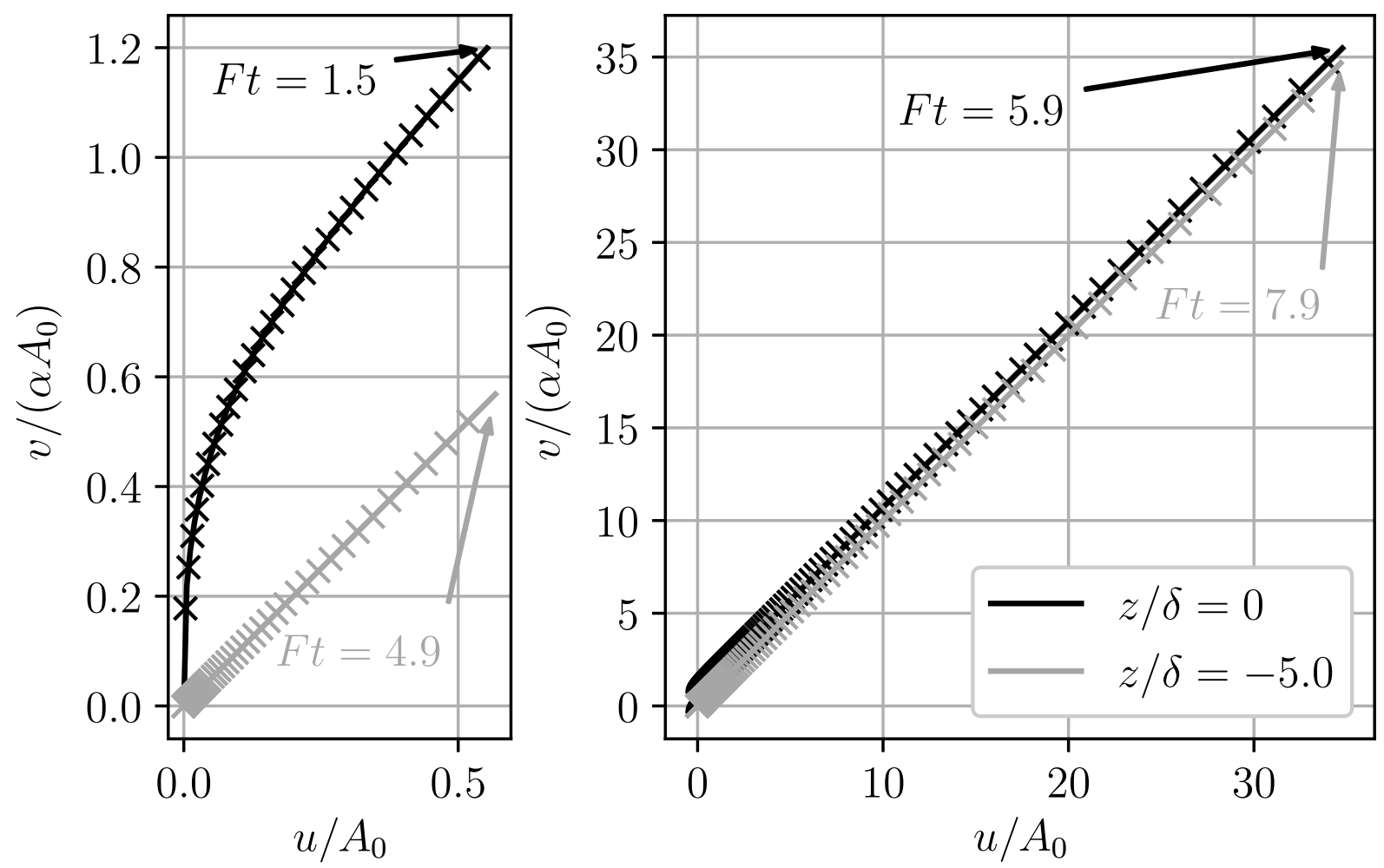

FIG. 5. Scaled hodographs at two different depths as time progresses. Left: short-term behavior. Right: long-term behavior. Annotated arrows indicate the time stamps on the last point of a given line. Solid lines are the theoretical prediction and crosses are the numerical simulation, with one cross displayed every time step. The axes are scaled equally, showing true angles in $(u, v / \alpha)$ coordinates.

\section{Transport}

Contrary to the EL case and its spiraling hodograph, the vertically integrated volume transport due to EII is mostly aligned with the direction of velocity field. When wind changes abruptly, we have, in EII coordinates,

$$
\left[\begin{array}{l}
M^{(U)} \\
M^{(V)}
\end{array}\right]=\delta \int_{-\infty}^{0}\left[\begin{array}{l}
U \\
V
\end{array}\right] \mathrm{d} Z^{\prime}=\frac{A_{0} \delta}{2}\left[\begin{array}{c}
\mathrm{e}^{\tau}-1 \\
1-\mathrm{e}^{-\tau}
\end{array}\right],
$$


or, in across- and along-front coordinates,

$$
\boldsymbol{M}=\left[\begin{array}{l}
M^{(u)} \\
M^{(v)}
\end{array}\right]=A_{0} \delta\left[\begin{array}{c}
\cosh \tau-1 \\
\alpha \sinh \tau
\end{array}\right],
$$

respectively. Also note that unlike $\sigma_{U}$, the growth rate of the mass transports reaches $F$ quickly, i.e., over a duration of $O\left(F^{-1}\right)$.

\section{DISCUSSIONS}

\section{A. Comparison with InI}

Unlike many instabilities, the features of EII did not reveal themselves via traditional normal mode analysis. That is, while our initial flow $\bar{v}, \bar{p}$ was the solution of a geostrophic balance and appropriate wind stress at the surface, we did not superpose wave-like perturbations, which is traditionally done, for example for InI, to compute linear growth rate and determine whether the perturbations may grow. Instead, we added a finite deviation from the top boundary condition by adding some wind stress. In this case, the initial "kick" did not consist of instantiating perturbations in the volume that may or may not grow, but resulted from a finite, albeit persistent, change in boundary conditions, which in turn created deviations that may or may not have grown. We also recall that this "kick" can be any change in wind stress, namely, an increase, a decrease, or a change in direction. We have demonstrated that for Ro $<-1$, once triggered, the induced flow deviation eventually grows in the runaway fashion that is the hallmark of hydrodynamic instabilities, and extracts its energy from the lateral shear of the flow at a rate that eventually converges to that of InI. This similarity in phenomenology, especially when compared to the finite nature of the boundary perturbations, lead us to classify this phenomenon as a hydrodynamic instability.

EII exhibits further differences with InI. For InI, $-L S P$ is the sole source of energy of the unstable perturbations. Velocities grow as part of spatially global wave-like modes, as opposed to the local (i.e., stress-driven) nature of EII expansion. In InI, the viscous flux divergence $\Phi_{z}$ and kinetic energy dissipation $\varepsilon$ have passive roles. That is, they are enhanced where InI creates stronger vertical shear, and decrease the growth rate everywhere by a constant amount $\nu m^{2}$, where $m$ is the vertical wavenumber of the growing mode. Moreover, because $-L S P$ is not scale-selective, InI occurring in a comparable horizontally 
invariant domain tends to select larger scales to minimize the importance of viscous effects, while the vertical scale of the EII flow constantly increases with $\sqrt{\nu t}$.

Viscosity induces another major practical difference between InI and EII, namely that a large value of (eddy) viscosity can only prevent the former from growing, while it can aid the latter's expansion. Indeed, $\mathrm{InI}$ modes grow at a rate $F-\nu m^{2}$, and viscosity's only role is that of damping and scale selection. In EII, however, a larger viscosity has two consequences: (1) it speeds up the vertical propagation of EII via a larger $\delta$, and (2) it decreases its magnitude since $A_{0} \propto \nu^{-1 / 2}$. However, because EII grows fast during VIP, we can reasonably anticipate it to rapidly become detectable even in a highly turbulent environment, and to impart its signature at depth. Therefore, we argue that regardless of the value of eddy viscosity, EII is likely to always manifest itself, be it as an intense, nearsurface current, or as a slower, slab-like motion of a significant vertical fraction of the front, or as some intermediate behavior.

One point of convergence between InI and EII refers to the $45^{\circ}$-angle in stretched coordinates between mean and EII flow. Recall however that we cautioned in $\S \amalg I I B$ against likening it to the surface deviation from the wind direction of the EL solution. On the other hand, a volume disturbance triggering InI would also induce flow that quickly aligns with the same angle as that of EII, by virtue of eqns. (9), which both EII and InI share.

\section{B. Finite width of currents}

As with all instabilities, EII induces a flow that will mix stable and unstable fluid, eventually extinguishing itself. Our solution does not include this effect because we kept Ro, i.e., $\zeta$, constant, effectively providing an endless supply of unstable fluid. In an actual front however, $\zeta$ varies in space. In that case, $M^{(u)}$, the cross-jet volume flux induced by EII, will eventually provoke its extinction: the front is indeed surrounded by stable, Ro $>-1$ fluid, which would cap the unstable region and stop EII from growing any further.

Furthermore, EII will grow at different rates depending on the location within a front because Ro varies in space. As a consequence, a horizontal velocity divergence $u_{x}$ will develop, compensated by a vertical velocity divergence $w_{z}$, a process called Ekman pumping for ELs. We can compute the vertical velocity $w_{\infty}$ well below the region where EII occurs by vertically integrating the mass continuity equation, yielding $w_{\infty}=-M_{x}^{(u)}$. A compre- 
hensive treatment of the corresponding "Ekman-inertial pumping" will require at least a two-dimensional study, and its complexity will be compounded by the fact that $\mathrm{Ro}=O(1)$, meaning that $x$ - and $z$-directions will be strongly coupled [26]. We defer this study to future work.

\section{CONCLUSIONS}

Oceanic flows with anticyclonic vertical vorticity that over-compensates planetary vorticity (i.e., Ro $<-1$ ), are unstable to perturbations in surface boundary conditions. These perturbations rapidly propagate down via tangential viscous stress, at a rate that far supersedes that of InI if the wind changes rapidly enough, at least initially so. We called this regime "Viscous-Inertial Peeling". After the instability is "primed" by the viscous stress however, the instability behaves like a slightly modified InI. In the simplest possible mathematical description we can make of it, namely, a columnar model, the vertical shear, compensated for inertial exponential growth, essentially follows a Rayleigh problem, and inherits its infinite initial growth rate. Assuming an abrupt change in wind conditions allowed us to write closed forms for the solutions, and therefore to make some of this behavior more explicit. After VIP, mass transport grows exponentially, at a rate $F$.

This instability not only shares several of its features with InI, but the behaviour of viscous stresses inevitably brings up features, more common to an Ekman spiral, superposed with inertial oscillations. In fact, we mirror our derivation with that for the Ro $>-1$ case in the Appendix, which highlights striking similarities, and which prompted us to call this instability "Ekman-Inertial Instability". In particular, the viscous top-down momentum flux is common to both, and its formal ties with Stoke's first problem provides EII with a fast growth rate that may makes it competitive with other instabilities such as InI, its baroclinic generalizations within the framework of centrifugal or symmetric instability, or baroclinic instability.

Whether this instability is novel or a mere flavor of $\mathrm{InI}$ is up for interpretation. More important however is to recognize EII's peculiar behavior, which may manifest itself in peculiar ways in actual ocean fronts. The geostrophic balance above neglects viscous diffusion of momentum, which we justify by assuming that the spatial scales of the geostrophic flow are too large for it to act over the time scales of EII. Investigating more realistic, i.e., two-and 
three-dimensional configurations, will be the topic of future work. The points we raised in $\S[\mathrm{IIC}$ would be a good start, which would raise new questions. In particular, how EII behaves in the presence of vertical and cross-jet buoyancy variations promises interesting discussions. Our one-dimensional model can easily incorporate an evolution equation for the buoyancy $b$, namely,

$$
b_{t}-u \bar{b}_{x}=\kappa b_{z z},
$$

where $\bar{b}$ is the mean buoyancy field and $\kappa$ is the buoyancy diffusivity coefficient. For EII to be an instability of the geostrophic flow, thermal wind balance has to apply, namely, $\bar{b}_{x}=\bar{v}_{z} / f$. In that case, in order for the initial condition to be a steady solution of the equations of motion, the wind stress has to be $\left.T^{y}(t<0) \equiv \rho \nu f \bar{b}_{x}\right|_{z=0}$, i.e., it has to maintain the surface thermal wind shear, as in previous studies [e.g., 27]. In our one-dimensional model still, $b$ does not feed back into the momentum equations (4). Therefore, EII can advect water masses of different densities across the front, which could directly modify the potential energy of a density front. Grisouard [26] had observed that with similar boundary conditions, and contrary to predictions from symmetric instability theory, a horizontal flow was advecting buoyancy laterally immediately under the surface and extracting potential energy from the front. Moreover, minimal potential energy exchanges were found between front and fluctuations when the minimum anticyclonic Rossby number was large, which would have suppressed EII, and the Richardson number of the thermal wind shear was small, which would have favored symmetric instability. At the time, these behaviors had no complete explanations. In light of our results however, they were consistent with EII out-competing symmetric instability whenever Ro was sufficiently anticyclonic.

Finally, stability of EII to along-jet and other three-dimensional disturbances such as convection, surface wave effects [28, 29] or non-traditional effects [30] should be investigated. Also, the simple viscosity we have used here is only a placeholder for turbulent momentum diffusion, whose effects are far from understood [e.g., 31, 32]. We could also include a more complete description of the competition with the transient growth of centrifugal, symmetric and/or baroclinic instability [33]. One possible avenue to is to compare EII with the large eddy simulations of frontal evolution [34, 35]. In particular, Skyllingstad et al. 34] simplified the dynamics of an unstable submesoscale density filament subjected to varying winds by neglecting all lateral geostrophic gradients and only retaining lateral buoyancy gradients. In their model, sufficiently strong EL and thermal wind shears couple to give rise to an 
"Ekman instability". On the contrary, EII requires a sufficiently strong anticyclonic shear, and it not directly affected by lateral buoyancy gradients, as we mentioned previously. In a follow-up work [36], the authors add sharp lateral gradients to their front, but do not include considerations about the Rossby number. It might be worthwhile to combine both descriptions to obtain more a complete description of submesoscale instabilities.

\section{ACKNOWLEDGMENTS}

We acknowledge the support of the Natural Sciences and Engineering Research Council of Canada (NSERC) [RGPIN-2015-03684], and of the Canadian Space Agency [14SUSWOTTO]. We acknowledge fruitful discussions with James C. McWilliams, which started during the Kavli Institute of Theoretical Physics program on Planetary Boundary Layers in Atmospheres, Oceans, and Ice on Earth and Moons (supported by the National Science Foundation under Grant No. NSF PHY-1748958), with Francis Poulin, and with Leif N. Thomas. We also acknowldege invaluable input from an anonymous reviewer.

\section{Appendix: Comparison with the establishment of an Ekman spiral}

When Ro $>-1$, re-defining $F=\beta f$, with $\beta=\sqrt{1+\mathrm{Ro}}$, better reveals the set-up of an EL. In doing so, eqns. (8)-(9) apply, albeit with the new definition of $F$. Note that we do not need to solve for both $U$ and $V$ anymore, since $u$ and $v$ derive from the real and imaginary parts of either of them. In line with the traditional presentation of ELs, we solve for $\tilde{V}=u+i v / \beta$ and introduce the counter-rotated field $\tilde{V}^{\ddagger}=\tilde{V} \mathrm{e}^{i \tau}$ to obtain the same diffusion equations such as the one in (10), and the counter-rotated boundary condition $\left.\tilde{V}_{Z}^{\ddagger}\right|_{Z=0}=i A(\tau) \mathrm{e}^{i \tau}$, with $A=\left.v_{z}\right|_{z=0} / \beta$. The solution is formally identical to eqn. (13), with the exception of $i \mathrm{e}^{-i \theta}$ replacing $\mathrm{e}^{-\theta}$. When surface boundary conditions change abruptly,

$$
\tilde{V}=\frac{A_{0} \mathrm{e}^{i \pi / 4}}{2 \sqrt{2}}\left[\mathrm{e}^{Z \sqrt{2 i}} \operatorname{erfc}\left(-\sqrt{i \tau}-\frac{Z}{\sqrt{2 \tau}}\right)-\mathrm{e}^{-Z \sqrt{2 i}} \operatorname{erfc}\left(\sqrt{i \tau}-\frac{Z}{\sqrt{2 \tau}}\right)\right] .
$$

As $\tau \rightarrow \infty, \tilde{V} \rightarrow A_{0} \mathrm{e}^{Z+i(\pi / 4+Z)} / \sqrt{2}$, which is the classical Ekman spiral solution. To obtain this result, we used the identities

$$
\frac{\mathrm{e}^{i \pi / 4}}{\sqrt{2}} \operatorname{erf}(\sqrt{i \tau})=\mathrm{S}(\sqrt{\hat{\tau}})+i \mathrm{C}(\sqrt{\hat{\tau}}) \rightarrow \frac{1+i}{2},
$$


where $\mathrm{S}$ and $\mathrm{C}$ are the normalized Fresnel integrals, $\hat{\tau}=2 \tau / \pi$, and the last arrow implies $\lim _{\hat{\tau} \rightarrow \infty}$.

At the surface,

$$
\left.\tilde{V}\right|_{Z=0}=A_{0}[\mathrm{~S}(\sqrt{\hat{\tau}})+i \mathrm{C}(\sqrt{\hat{\tau}})] .
$$

For $\tau \ll 1, \mathrm{C}(\sqrt{\hat{\tau}}) \approx \sqrt{\hat{\tau}}$, i.e., exhibits a growth rate singularity, similar to that of EII. In the other limit $\tau \gg 1, \mathrm{C}(\sqrt{\hat{\tau}})-1 / 2 \approx \sin \tau / \sqrt{2 \pi \tau}$, with $\mathrm{S}$ behaving similarly. That is, the convergence to the EL solution manifests itself as near-inertial, or near- $F$ frequency, pseudooscillations. Note that their envelope decays as $1 / \sqrt{2 \pi \tau}$, identical to that of $\sqrt{2 / \pi} \mathrm{D}(\sqrt{\tau})$, the compensated EII magnitude. The time evolution of the surface hodograph resembles that of a Cornu spiral, albeit one that converges more slowly towards its attractor and with a constant quasi-frequency $F$.

Like EII, this solution highlights two phases: first, that of a rapid adjustment (singular growth rate), followed by a slow $\left(\sim \tau^{-1 / 2}\right)$ and oscillatory convergence towards constant values $u / A_{0}=v /\left(\beta A_{0}\right)=-1 / 2$, which is the surface expression of the EL. These two phases are of course the stable counterparts to EII's VIP and InVI stages. In fact, because we defined VIP as the phase during which rotation has not affected the motion yet, it appears natural that VIP is shared by both EII and EL.

Contrary to EII however, a wind disturbance of arbitrary orientation corresponds to a surface boundary condition for $\tilde{V}_{z}$ that is not purely imaginary, and whose phase encodes the disturbance direction. As a result, the orientation of $u$ and $v$ is with respect to the wind direction, not the mean flow.

[1] G. K. Vallis, Atmospheric and Oceanic Fluid Dynamics (Cambridge University Press, Cambridge, 2017).

[2] V. W. Ekman, On the influence of the earth's rotation on ocean-currents., Arkiv för Matematik, Astronomi och Fysik 2, 1 (1905).

[3] J. O. Wenegrat and L. N. Thomas, Ekman transport in balanced currents with curvature, Journal of Physical Oceanography 47, 1189 (2017).

[4] M. E. Stern, Interaction of a uniform wind stress with a geostrophic vortex, Deep Sea Research and Oceanographic Abstracts 12, 355 (1965) 
[5] P. P. Niiler, On the Ekman divergence in an oceanic jet, Journal of Geophysical Research 74, 7048 (1969).

[6] Y. Morel and L. N. Thomas, Ekman drift and vortical structures, Ocean Modelling 27, 185 $(2009)$.

[7] D. G. Dritschel, N. Paldor, and A. Constantin, The Ekman spiral for piecewise-uniform diffusivity, Ocean Science Discussions in review, 10.5194/os-2020-31 (2020).

[8] V. I. Shrira and R. B. Almelah, Upper-ocean Ekman current dynamics: a new perspective, Journal of Fluid Mechanics 887, A24 (2020),

[9] J. O. Wenegrat and M. J. McPhaden, Wind, Waves, and Fronts: Frictional Effects in a Generalized Ekman Model*, Journal of Physical Oceanography 46, 371 (2016).

[10] L. N. Thomas, A. Tandon, and A. Mahadevan, Submesoscale processes and dynamics, in Ocean Modeling in an Eddying Regime, Geophysical Monograph Series, Vol. 177, edited by M. W. Hecht and H. Hasumi (American Geophysical Union, Washington, D. C., 2008) pp. $17-38$.

[11] J. C. McWilliams, Submesoscale currents in the ocean, Proceedings of the Royal Society A: Mathematical, Physical and Engineering Science 472, 20160117 (2016).

[12] A strict definition of submesoscale flows would also include a Richardson number that is order one, i.e., vertical geostrophic velocity gradients that are comparable to the buoyancy frequency of the density stratification. However, we will mostly ignore such effects.

[13] P. Klein and G. Lapeyre, The Oceanic Vertical Pump Induced by Mesoscale and Submesoscale Turbulence, Annual Review of Marine Science 1, 351 (2009),

[14] M. Lévy, P. J. S. Franks, and K. S. Smith, The role of submesoscale currents in structuring marine ecosystems, Nature Communications 9, 4758 (2018).

[15] A. de Verneil, P. J. S. Franks, and M. D. Ohman, Frontogenesis and the creation of fine-scale vertical phytoplankton structure, Journal of Geophysical Research: Oceans , 2018JC014645 (2019).

[16] J. O. Wenegrat, L. N. Thomas, J. Gula, and J. C. McWilliams, Effects of the submesoscale on the potential vorticity budget of Ocean Mode Waters, Journal of Physical Oceanography 48, 2141 (2018).

[17] Z. Su, J. Wang, P. Klein, A. F. Thompson, and D. Menemenlis, Ocean submesoscales as a key component of the global heat budget, Nature Communications 9, 775 (2018). 
[18] R. Ferrari and C. Wunsch, Ocean Circulation Kinetic Energy: Reservoirs, Sources, and Sinks, Annual Review of Fluid Mechanics 41, 253 (2009).

[19] J. C. McWilliams, E. Huckle, J.-H. Liang, and P. P. Sullivan, The Wavy Ekman Layer: Langmuir Circulations, Breaking Waves, and Reynolds Stress, Journal of Physical Oceanography 42, 1793 (2012).

[20] J. C. McWilliams, J. Gula, M. J. Molemaker, L. Renault, and A. F. Shchepetkin, Filament Frontogenesis by Boundary Layer Turbulence, Journal of Physical Oceanography 45, 1988 $(2015)$.

[21] M. N. Crowe and J. R. Taylor, The evolution of a front in turbulent thermal wind balance. Part 1. Theory, Journal of Fluid Mechanics 850, 179 (2018).

[22] T. W. N. Haine and J. Marshall, Gravitational, Symmetric, and Baroclinic Instability of the Ocean Mixed Layer, Journal of Physical Oceanography 28, 634 (1998).

[23] B. Cushman-Roisin and J.-M. Beckers, Introduction to Geophysical Fluid Dynamics, 2nd ed. (Academic Press, 2011).

[24] K. J. Burns, G. M. Vasil, J. S. Oishi, D. Lecoanet, and B. P. Brown, Dedalus: A flexible framework for numerical simulations with spectral methods, Physical Review Research 2, 023068 (2020).

[25] See supplemental material at https://github.com/ngrisouard/ Ekman-Inertial-Instability.

[26] N. Grisouard, Extraction of Potential Energy from Geostrophic Fronts by Inertial-Symmetric Instabilities, Journal of Physical Oceanography 48, 1033 (2018).

[27] J. R. Taylor and R. Ferrari, On the equilibration of a symmetrically unstable front via a secondary shear instability, Journal of Fluid Mechanics 622, 103 (2009).

[28] J. C. McWilliams and B. Fox-Kemper, Oceanic wave-balanced surface fronts and filaments, Journal of Fluid Mechanics 730, 464 (2013).

[29] J. C. McWilliams, Surface wave effects on submesoscale fronts and filaments, Journal of Fluid Mechanics 843, 479 (2018).

[30] V. Zeitlin, Letter: Symmetric instability drastically changes upon inclusion of the full Coriolis force, Physics of Fluids 30, 061701 (2018).

[31] P. P. Sullivan and J. C. McWilliams, Frontogenesis and frontal arrest of a dense filament in the oceanic surface boundary layer, Journal of Fluid Mechanics 837, 341 (2018). 
[32] V. Verma, H. T. Pham, and S. Sarkar, The submesoscale, the finescale and their interaction at a mixed layer front, Ocean Modelling 140, 101400 (2019).

[33] V. E. Zemskova, P.-Y. Passaggia, and B. L. White, Transient energy growth in the ageostrophic Eady model, Journal of Fluid Mechanics 885, A29 (2020).

[34] E. D. Skyllingstad, J. Duncombe, and R. M. Samelson, Baroclinic Frontal Instabilities and Turbulent Mixing in the Surface Boundary Layer, Part II: Forced Simulations, Journal of Physical Oceanography , 16 (2017).

[35] H. T. Pham and S. Sarkar, Ageostrophic Secondary Circulation at a Submesoscale Front and the Formation of Gravity Currents, Journal of Physical Oceanography 48, 2507 (2018).

[36] E. D. Skyllingstad and R. M. Samelson, Instability Processes in Simulated Finite-Width Ocean Fronts, Journal of Physical Oceanography 50, 2781 (2020). 